\title{
Electroosmotic flow of a power-law fluid through an asymmetrical slit microchannel with gradually varying wall shape and wall potential
}

\author{
Cheng Qi, Chiu-On $\mathrm{Ng}^{1}$ \\ Department of Mechanical Engineering, The University of Hong Kong, \\ Pokfulam Road, Hong Kong
}

\begin{abstract}
This study aims to investigate electroosmotic flow of a power-law fluid through a slit channel with walls asymmetrically patterned with periodic variations in shape and zeta potential. On taking into account the near-wall depletion layer, the present problem is simplified on the basis of the lubrication approximation, and through the use of the Helmholtz-Smoluchowski slip boundary condition. Nonlinear equations are to be solved for two unknown functions of axial dependence, one being the induced pressure gradient, and another being an undetermined stress component. An efficient numerical scheme is devised in this work to solve the nonlinear problem. Results are generated to check whether the principle of linear superposition of forces, for electrokinetic flow of a nonNewtonian fluid in the presence of a Newtonian depletion layer, is still applicable to flow in an asymmetric channel. It is also found that phase shifts between the geometrical and electric potential patterns on the two walls may lead to qualitatively disparate effects, depending on the power-law behavior index of the fluid and the applied pressure gradient.
\end{abstract}

Keywords: electroosmotic flow; power-law fluid; lubrication approximation.

\footnotetext{
${ }^{1}$ Corresponding author. Tel: (852) 2859 2622; Fax: (852) 28585415; E-mail address: cong@hku.hk (C.-O. $\mathrm{Ng})$.
} 


\section{Introduction}

In electrokinetic transport, the processes are usually so slow that a flux may depend in a linear manner on its conjugate as well as nonconjugate forces [1]. For example, the fluid flow can be driven not only by a pressure gradient, but also by an electric potential gradient, both through a linear relationship. Typically, electrokinetic fluxes are a linear combination of the components due individually to the hydrodynamic or electric driving forces. All such linear relationships, also known as Onsager relations, are applicable to materials with linear response, such as Newtonian fluid.

Electrokinetic transport of a non-Newtonian fluid does not follow a linear law in general, mainly because of the nonlinear rheological constitutive relation. Nonetheless, Berli and Olivares [2] have shown that, on taking into account the wall depletion effect, electroosmotic flow (EOF) of non-Newtonian fluids is also expressible by a linear superposition of the components due separately to the hydrodynamic and electric forcings. They found that the nonlinear effects arising from the shear-dependent viscosity are limited to the pressure-driven component of flow, and the Onsager reciprocity, which means the equality of the nonconjugate streaming coefficients, is also complied with.

For complex fluids, such as colloids, emulsions and polymeric solutions, there usually exists a thin depletion layer near a non-sorbing wall $[3,4]$. The depletion layer is so called because it is depleted of the macromolecules that give rise to the nonlinear behavior of the complex fluid. It is essentially a layer of a thickness comparable to the radius of gyration of the macromolecules. In the absence of macromolecules, the fluid in the depletion layer is actually the Newtonian solvent of the solution. Assuming that the depletion layer is much thicker than the electric double layer (EDL), Berli and Olivares [2] put forward a two-zone model for EOF of non-Newtonian fluids in a microchannel. The core zone is a region filled with a non-Newtonian fluid forced directly by pressure gradient only. This core region is electrically neutral since the EDL is completely screened in the depletion layer. The near-wall zone is of course the depletion layer, which is Newtonian, and is subjected to both hydrodynamic and electric forces. By virtue of this zonal separation of the fluid behaviors as well as the forcings, Berli and Olivares [2] obtained force-flux relations that are a linear combination of 
two components, one associated with the pressure difference only, and the other associated with the electric potential difference only. Unlike the Newtonian case, the hydrodynamic conductance based on this two-zone model is not a constant, but a nonlinear function of the pressure difference.

The channels considered by Berli and Olivares [2] are uniform microchannels with either plane symmetry or axisymmetry. The flow they have considered is hence strictly onedimensional and symmetrical flow through a parallel-plate or circular channel with a constant cross section and a constant zeta potential. One would ask a question: can the generalized force-flux relations found by Berli and Olivares [2] still be valid if the channels are non-uniform or asymmetric?

Whether the linear superposition of forces is applicable to non-Newtonian EOF in a nonuniform channel has been examined by Ng and Qi [5]. These authors presented a model for EOF of a power-law fluid through a non-uniform slit channel, which has the cross section as well as the wall potential to vary slowly with axial position, but with mid-plane symmetry. They found that such axial non-uniformities, resulting in a non-parallel flow, will invalidate the linear superposition of forces to drive the flow of a non-Newtonian fluid, even when the wall depletion effect is taken into account. For EOF of a non-Newtonian fluid through a non-uniform channel, the flow rate due to the combined action of applied pressure gradient and electric field is not equal to the sum of the flow rates due separately to these forces.

The present study aims to extend the work of $\mathrm{Ng}$ and Qi [5] in an attempt to further look into EOF of a non-Newtonian fluid through a channel that is not only non-uniform in the axial direction, but also asymmetric about the mid-plane of the channel. The primary objective is to examine the effect of flow symmetry on the validity of the generalized forceflow relation. We shall seek an answer to the question posed above by considering combined pressure-driven and electroosmotic flow of a power-law fluid through a slit channel without plane symmetry. On the one hand, losing the symmetry condition will make the problem more difficult to solve. Complication arises from the need to identify regions of positive or negative stress in order to apply the power-law rheological model. Therefore, another objective of this study is to develop an efficient numerical solution method to handle this kind of nonlinear problem. On the other hand, on relaxing the symmetry requirement, we 
may investigate effects on the flow due to the phase shift between the patterns on the two walls of the channel. Such an investigation is not possible for flow in a symmetric channel. Therefore, the third objective of this study is to find out how the phase difference between the geometrical and electric potential distribution patterns on the opposite walls of the channel may have disparate effects on the flow, depending on the rheology of the fluid.

The present problem is basically similar in mathematical formulation to that presented by $\mathrm{Ng}$ and Qi [5]. The key aspects of the problem formulation are summarized as follows. First, the power-law model is chosen to describe the non-Newtonian behavior of the fluid. EOF of power-law fluid has been extensively studied in recent years in the context of electrokinetic transport in microfluidic devices [6-29]. Most of these existing studies are, however, limited to flow in relatively simple configurations, e.g., channels of uniform cross section, constant zeta potential distribution, and so on. Also, some experimental attempts have been made to investigate EOF under the effect of surface heterogeneities. Again, the majority of these previous studies are for simple pattern configurations (e.g., either surface charge patterning on channel walls [30-33] or patterned blocks in a channel [34]), or limited to Newtonian fluid flows [30-34]. To date, little research has been conducted to examine in particular EOF of a power-law fluid in a channel with asymmetrical, non-uniformly shaped and charged walls. This key essence distinguishes the present study from the previous ones. Second, the two-zone model put forward by Berli and Olivares [2] is adopted. Near the wall is a very thin depletion layer, yet thick enough to completely cover the EDL. Hence, the electric force is applied only to the Newtonian fluid in the depletion layer. The core region, which is occupied by a powerlaw bulk fluid, is electro-neutral. Although the electric body force is zero in the core region, the bulk fluid is driven by two forces: the direct forcing of pressure gradient, and the indirect forcing of electrokinetics through the continuity of velocity on the interface between the two zones. Third, the variations of flow in the axial direction are assumed to be so mild that the lubrication approximation $[35,36]$ can be applied to the present problem. This will simplify the governing equations to a quasi-one-dimensional form, significantly reducing the numerical efforts required to solve the problem. Fourth, to further simplify the problem, we shall follow MacInnes et al. [37], and Zimmerman et al. [38] to adopt the Helmholtz-Smoluchowski (HS) slip boundary condition [39] instead of resolving the EDL directly for the electrokinetic force. 
We assume that the channel height, which may be of the order of $100 \mu \mathrm{m}$, is much larger than the depletion layer thickness (say, $100 \mathrm{~nm}$ ), which is in turn much larger the EDL thickness (say, $10 \mathrm{~nm}$ ). Using the HS slip condition to represent the electrokinetic forcing, the analysis can be performed in a simplified manner focusing on the bulk fluid flow only. Berli and Olivares [2] have theoretically shown that in the limiting case of an infinitesimally thin depletion layer the electrokinetic action of the EDL on the bulk fluid will asymptotically reduce to the electroosmotic slip boundary condition.

\section{The Problem}

Following $\mathrm{Ng}$ and Qi [5], we examine in this study combined pressure-driven and electroosmotic flow of a power-law fluid through a slit channel bounded by two nearly parallel walls, where the channel height as well as the wall potential may vary slowly and periodically with axial position. In the previous study [5], the patterns on the two walls are assumed to be aligned so that the flow is symmetrical about the centerline. In the present study, this assumption is dropped. Here, we allow a phase difference between the patterns on the two walls: the geometrical as well as the electric potential patterns on one wall may not be aligned with those on the other wall. On relaxing this requirement of wall alignment, the flow in the present problem is in general asymmetrical about the channel centerline, and therefore we need to solve the problem across the entire section of the channel. Figure 1 shows a definition sketch of our problem, where the axial and transverse coordinates are denoted by $(x, y)$, and the channel centerline is along $y=0$. Channel non-uniformities are in the form of variations in both the wall potential and wall shape: $\zeta_{1,2}(x)$ are the upper/lower wall (or zeta) potentials, and $h_{1,2}(x)$ are the distances from the centerline of the upper and lower walls. They are all periodic functions of $x$ with the same wavelength $L$. With an average channel height of $2 h_{0}$, the upper wall is located at $y=h_{1}(x)=h_{0}+\Delta h_{1}(x)$, while the lower wall is positioned at $y=-h_{2}(x)=-h_{0}-\Delta h_{2}(x)$, where $\Delta h_{1,2}$ are the undulations of the upper/lower walls. These undulations should have an amplitude less than half the average channel height: $\left|\Delta h_{1,2}\right|<h_{0}$. The present problem reduces to the previous problem by $\mathrm{Ng}$ and Qi [5] under the particular conditions: $\zeta_{1}=\zeta_{2}$ and $h_{1}=h_{2}$. 
The present problem is to be formulated based on the lubrication approximation, which is valid when two basic conditions are met $[5,35]$. The first condition, which is a geometrical condition, is that the lengthscale for variations of flow in the axial direction is much longer than that in the transverse direction. In this regard, we assume that the ratio of channel height to pattern wavelength, $h_{0} / L$, is a very small parameter. By virtue of this assumption, the spatial gradients of flow quantities in the axial direction will be much smaller than the counterparts in the transverse direction. As a result, the flow becomes a nearly unidirectional or parallel flow. The second condition, which is a dynamical condition, is that the Reynolds number is order unity or smaller. This condition leads to sub-dominant inertia terms in the momentum equations.

The lubrication approximation will result in an axial momentum equation that looks like the one for a strictly one-dimensional flow. The key difference is that the pressure gradient is now an unknown function of $x$. The pressure, which is induced internally so as to satisfy continuity of flow through a non-uniform channel, is a nonlinear function of the axial non-uniformities. For Newtonian fluid flows, this induced pressure is usually found by axial averaging. For non-Newtonian fluid flows, the task of determining the induced pressure can be more complicated, mainly because of the nonlinear relationship between the shear stress and shear rate. As is shown below, without the symmetry condition, the sectional shear stress distribution will admit an undetermined stress component when the momentum equation is integrated with respect to $y$. This imposes further difficulty in solving for the induced pressure. This is the main challenge of the present study, and also distinguishes the present study from the previous study [5].

The bulk fluid is assumed to be a power-law fluid, for which the constitutive equation under simple shear is as follows:

$$
\tau=\mu\left|\frac{\partial u}{\partial y}\right|^{n-1} \frac{\partial u}{\partial y}= \begin{cases}\mu\left(\frac{\partial u}{\partial y}\right)^{n} & \text { for } \frac{\partial u}{\partial y} \geq 0 \\ -\mu\left(-\frac{\partial u}{\partial y}\right)^{n} & \text { for } \frac{\partial u}{\partial y}<0\end{cases}
$$

where $\tau$ is the shear stress, $u$ is the axial velocity, and $\mu$ and $n$ are the flow consistency and flow behavior index of the fluid, respectively. The value of $n$ determines the behaviors of the fluid: $n<1,=1,>1$ correspond to shear-thinning, Newtonian, and shear-thickening behaviors, respectively. To facilitate deduction, the above constitutive equation is rewritten 
as follows:

$$
\frac{\partial u}{\partial y}=\mu^{-\frac{1}{n}}|\tau|^{\frac{1}{n}-1} \tau= \begin{cases}\left(\frac{\tau}{\mu}\right)^{\frac{1}{n}} & \text { for } \tau \geq 0 \\ -\left(-\frac{\tau}{\mu}\right)^{\frac{1}{n}} & \text { for } \tau<0\end{cases}
$$

Two conditions involving a sharp contrast between three thicknesses are assumed here: the EDL is much thinner than the near-wall depletion layer, while the depletion layer is much thinner than the channel height. Fluid in the depletion layer, owing to the absence of the inclusion, is much less viscous than the bulk fluid and is essentially the Newtonian solvent of the power-law fluid. Hence, the first condition is to ensure that the electrokinetic body force acts only on the Newtonian fluid adjacent to the wall. Further under the second condition, the electrokinetic forcing may, asymptotically and effectively, reduce to a Helmholtz-Smoluchowski (HS) slip velocity on each of the two walls:

$$
\begin{gathered}
u=-\frac{\epsilon E_{x}}{\mu_{s}} \zeta_{1}(x) \quad \text { at } y=h_{1}(x), \\
u=-\frac{\epsilon E_{x}}{\mu_{s}} \zeta_{2}(x) \quad \text { at } y=-h_{2}(x),
\end{gathered}
$$

where $\epsilon$ and $\mu_{s}$ are the permittivity and dynamic viscosity of the Newtonian fluid in the depletion layer, and $E_{x}$ is the externally applied axial electric field. By virtue of the slow change in the wall topography (i.e., a very small steepness of the undulations), we may apply the HS slip conditions as if the walls were perfectly flat.

Under the lubrication approximation, the Cauchy momentum equation simplifies to

$$
0=K+\frac{\partial \tau}{\partial y}
$$

where $K(x)=-\mathrm{d} p / \mathrm{d} x$ is the pressure gradient, which consists of a known constant component (i.e., the applied pressure gradient) and an undetermined periodic component (i.e., the internally induced pressure gradient), given that the wall potentials and heights vary periodically with axial position.

Let us now introduce the following normalized variables (distinguished by overhead carets):

$$
\left.\begin{array}{rl}
\hat{x}=x / L, \quad\left(\hat{y}, \hat{h}_{1,2}\right) & =\left(y, h_{1,2}\right) / h_{0}, \quad \hat{u}=u / u_{0}, \\
\hat{\zeta}_{1,2}=\zeta_{1,2} / \zeta_{0}, \quad \hat{\tau} & =\tau / \tau_{0}, \quad \hat{K}=K /\left(\tau_{0} / h_{0}\right),
\end{array}\right\},
$$


where $u_{0}=-\epsilon E_{x} \zeta_{0} / \mu_{s}, \tau_{0}=\mu u_{0}^{n} / h_{0}^{n}$, and $\zeta_{0}$ is a characteristic scale for the wall zeta potential. Note that the velocity is normalized by a scale that is independent of $n$, which will allow us to compare flow rates of different $n$ in our later discussion.

In terms of the normalized variables, Eq. (2) reads as

$$
\frac{\partial \hat{u}}{\partial \hat{y}}=|\hat{\tau}|^{\frac{1}{n}-1} \hat{\tau}
$$

while the HS slip boundary conditions in Eqs. (3)-(4) become

$$
\begin{gathered}
\hat{u}=\hat{\zeta}_{1}(\hat{x}) \quad \text { at } \hat{y}=\hat{h}_{1}(\hat{x}), \\
\hat{u}=\hat{\zeta}_{2}(\hat{x}) \quad \text { at } \hat{y}=-\hat{h}_{2}(\hat{x}) .
\end{gathered}
$$

Integrating Eq. (7) with respect to $\hat{y}$, and using boundary condition (9), we get

$$
\hat{u}=\hat{\zeta}_{2}+\int_{-\hat{h}_{2}}^{\hat{y}}|\hat{\tau}|^{\frac{1}{n}-1} \hat{\tau} \mathrm{d} \hat{y} .
$$

Also, by boundary condition (8),

$$
\int_{-\hat{h}_{2}}^{\hat{h}_{1}}|\hat{\tau}|^{\frac{1}{n}-1} \hat{\tau} \mathrm{d} \hat{y}=\hat{\zeta}_{1}-\hat{\zeta}_{2}
$$

The volume flow rate through the channel can be obtained by integrating the velocity across the channel:

$$
\hat{q}=\int_{-\hat{h}_{2}}^{\hat{h}_{1}} \hat{u} \mathrm{~d} \hat{y}=\hat{\zeta}_{2}\left(\hat{h}_{1}+\hat{h}_{2}\right)+\int_{-\hat{h}_{2}}^{\hat{h}_{1}} \int_{-\hat{h}_{2}}^{\hat{y}}|\hat{\tau}|^{\frac{1}{n}-1} \hat{\tau} \mathrm{d} \hat{y} \mathrm{~d} \hat{y} .
$$

By continuity, $\hat{q}$ is independent of the axial position $\hat{x}$.

The dimensionless form of Eq. (5) is

$$
0=\hat{K}+\frac{\partial \hat{\tau}}{\partial \hat{y}}
$$

By periodicity, the induced pressure gradient has a zero mean. Hence, integrating the pressure gradient $\hat{K}=\hat{K}(\hat{x})$ axially over one wavelength should give the pressure drop $\Delta \hat{P}$ in unity length of the channel due to the applied pressure gradient:

$$
\int_{0}^{1} \hat{K} \mathrm{~d} \hat{x}=\Delta \hat{P}
$$

which amounts to the normalized applied pressure gradient. The applied pressure gradient is favorable, zero, or adverse when the net pressure drop $\Delta \hat{P}>0,=0,<0$, respectively. 
Integrating Eq. (13) with respect to $\hat{y}$ gives us an expression for the transverse shear stress distribution:

$$
\hat{\tau}(\hat{x}, \hat{y})=-\hat{K} \hat{y}+\hat{\tau}_{c}
$$

where $\hat{\tau}_{c}=\hat{\tau}_{c}(\hat{x})$ is a yet-to-be-determined periodic function of $\hat{x}$. Unless the flow is symmetrical about the centerline for which $\hat{\tau}_{c}=0$, the stress contains two undetermined components, $\hat{K}(\hat{x})$ and $\hat{\tau}_{c}(\hat{x})$, which are to be found simultaneously. This makes the present solution method more complicated than that in the symmetry case, as has been remarked above.

\section{Solution Methods}

We shall for simplicity omit the overhead carets of the dimensionless variables from here

on. The problem is now to determine, for given $\zeta_{1,2}(x), h_{1,2}(x)$ and $\Delta P$, the two spatial functions $\tau_{c}(x)$ and $K(x)$, and the flow rate $q$ that satisfy Eqs. (11), (12) and (14), in which the stress $\tau(x, y)$ is given by Eq. (15). The problem is in general highly nonlinear and can only be solved numerically. Analytical or simpler solution methods are available only for some special cases. The special cases, as well as the general numerical solution method, are detailed in the following sections.

\subsection{Newtonian limit}

The problem can be solved analytically for the case of a Newtonian fluid. For $n=1$, Eqs. (11), (12) and (15) give

$$
\begin{aligned}
K & =\frac{12}{\left(h_{1}+h_{2}\right)^{3}}\left[q-\frac{1}{2}\left(h_{1}+h_{2}\right)\left(\zeta_{1}+\zeta_{2}\right)\right], \\
\tau_{c} & =\frac{1}{2}\left(h_{1}-h_{2}\right) K+\left(\zeta_{1}-\zeta_{2}\right) /\left(h_{1}+h_{2}\right) .
\end{aligned}
$$

On applying Eq. (14) to Eq. (16), the flow rate $q$ can then be found as

$$
q=\left[\Delta P+6 \int_{0}^{1}\left(\zeta_{1}+\zeta_{2}\right)\left(h_{1}+h_{2}\right)^{-2} \mathrm{~d} x\right] /\left[12 \int_{0}^{1}\left(h_{1}+h_{2}\right)^{-3} \mathrm{~d} x\right] .
$$

Putting $q$ back to Eq. (16), which is then substituted into Eq. (17), we may determine the two functions $K(x)$ and $\tau_{c}(x)$. Note that for any $\zeta_{1,2}(x)$ and $h_{1,2}(x), q$ is always a linear 
combination of the components due separately to the electric and hydrodynamic forcings for a Newtonian fluid.

\subsection{Flat and uniformly charged walls}

If the walls are flat $\left(h_{1,2}=1\right)$ and uniformly charged such that the potentials $\zeta_{1,2}$ are constants (but $\zeta_{1}$ and $\zeta_{2}$ can be different constants), the problem can be solved readily when the applied pressure gradient is zero, $\Delta P=0$. Under these conditions, the pressure gradient is identically zero, $K=0$, by which the stress reduces to a constant $\tau=\tau_{c}$. From Eq. (11), we get

$$
\tau_{c}= \begin{cases}{\left[\left(\zeta_{1}-\zeta_{2}\right) /\left(h_{1}+h_{2}\right)\right]^{n}} & \text { for } \zeta_{1}>\zeta_{2}, \\ -\left[\left(\zeta_{2}-\zeta_{1}\right) /\left(h_{1}+h_{2}\right)\right]^{n} & \text { for } \zeta_{2}>\zeta_{1}\end{cases}
$$

Then, from Eq. (12),

$$
q=\frac{1}{2}\left(\zeta_{1}+\zeta_{2}\right)\left(h_{1}+h_{2}\right)
$$

which is independent of the power-law behavior of the fluid. If $n \neq 1, \zeta_{1} \neq \zeta_{2}$ and $\Delta P \neq 0$, the problem has to be solved numerically using the method described below in Section 3.4.

\subsection{Symmetrical flow}

When $\zeta_{1}(x)=\zeta_{2}(x)=\zeta(x)$ and $h_{1}(x)=h_{2}(x)=h(x)$, the flow is symmetrical about the centerline of the channel, by which $\tau_{c}=0$. The problem is then simplified to the one presented by $\mathrm{Ng}$ and Qi [5]. The flow rate $q$ can be found using an iterative method from

$$
\left(\frac{1+2 n}{2 n}\right)^{n} \int_{0}^{1}(q-2 \zeta h) \frac{|q-2 \zeta h|^{n-1}}{h^{1+2 n}} \mathrm{~d} x=\Delta P .
$$

Then, the pressure gradient $K(x)$ is given by

$$
K=\left(\frac{1+2 n}{2 n}\right)^{n} \frac{|q-2 \zeta h|^{n-1}}{h^{1+2 n}}(q-2 \zeta h) .
$$

In the limiting case of a strictly uniform channel, i.e., $\zeta$ and $h$ are pure constants, the flow rate is given by

$$
q=2 \zeta h \pm\left(\frac{2 n}{1+2 n}\right) h^{\frac{1+2 n}{n}}( \pm \Delta P)^{\frac{1}{n}}
$$


where the upper/lower signs are for positive/negative $\Delta P$, respectively. Note that in this particular case, even for a non-Newtonian fluid, the flow rate is a linear combination of the two components due separately to the electric and hydrodynamic forcings. As has been remarked earlier, this linearity is a result of directly using the HS slip condition on taking into account the near-wall Newtonian depletion layer. Further details about this symmetrical case can be found in $\mathrm{Ng}$ and Qi [5].

\subsection{Numerical method}

For cases other than those described above, the problem solving is more complicated arising from the need to solve some nonlinear equations for the variables $q, K(x)$ and $\tau_{c}(x)$. A trialand-error numerical method, the steps of which are outlined below, is adopted to solve the problem.

I. The axial domain, $0 \leq x \leq 1$, is uniformly divided into $N-1$ subintervals, whereby the domain is discretized by $N$ equally spaced grid points at $x=x_{i}=(i-1) /(N-1)$, where $i=1, \cdots, N$. The functions $K(x)$ and $\tau_{c}(x)$ are then represented by their discrete values at these grid points.

II. The process begins with a trial value of the flow rate $q$. For a given $q$, the values of the pressure gradient $K_{i}=K\left(x_{i}\right)$ and the stress component $\tau_{c i}=\tau_{c}\left(x_{i}\right)$ at the discrete points can be found through some relationships based on Eqs. (11) and (12). Further details are provided below, and in the appendix.

III. The net pressure drop is then found by integrating $K_{i}$ over the domain, which is performed numerically using Simpson's formula. This calculated pressure drop is to compare with the given pressure drop $\Delta P$ in order to satisfy Eq. (14). Unless the difference between these pressure drops are very small, we shall repeat Step II with an adjusted trial value of $q$.

IV. Steps II and III are to be repeated until the calculated pressure drop agrees with the given pressure drop within a certain numerical accuracy. 
The essence of Step II is to develop an efficient way to determine $K_{i}$ for a given $q$. The most challenging part is to handle the nonlinear term $|\tau|^{1 / n-1}$, which appears in the integrands in Eqs. (11) and (12). It is desirable if the sign of $\tau$ can be made definite when the integrations are performed. To this end, we need to consider various possible combinations of $K$ and $\tau_{c}$, which are the components of $\tau$, as given by Eq. (15). All these combinations, grouped under seven cases, are detailed in Table 1.

Let us first explain how Table 1 is constructed, before we explain how it may be used to solve for $K$. To facilitate the analysis, we have introduced in cases $1-6$, where $K \neq 0$, an auxiliary variable $z \equiv \tau_{c} / K$, by which the stress is expressible by $\tau=-K(y-z)$ for $-h_{2} \leq y \leq h_{1}$. We define the seven cases according to the values of $K$ and $z$. Cases 1-3, 4-6, and 7 are for $K>0, K<0$, and $K=0$, respectively. Furthermore, cases 1 and 4 are for $-h_{2} \leq z \leq h_{1}$, while cases 2 and 5 are for $z>h_{1}$, and cases 3 and 6 are for $z<-h_{2}$. Accordingly, the regions where $\tau$ is positive or negative may be determined for the individual cases, as given in the fourth column of Table 1. A known sign of $\tau$ in different regions will then enable the integration in Eq. (11) to be performed explicitly. The integral in this equation also determines the sign of $\zeta_{1}-\zeta_{2}$, as given in the fifth column of Table 1 . In cases 1 and 4, we need to further differentiate between three sub-cases, (a), (b) and (c), according to $\zeta_{1}-\zeta_{2}>0,=0$ or $<0$. The conditions in terms of $z$ giving rise to these sub-cases are also given in the table. Finally, based on Eq. (12), we may determine for each case or sub-case the range in which $q /\left(h_{1}+h_{2}\right)$ will lie, as given in the sixth column of Table 1 . We leave all the mathematical details to the appendix.

Note that the seven cases given in Table 1 are mutually exclusive, and the union of them covers all the possible values of the inputs and outputs. In other words, for any given $q$ and local values of $h_{1}, h_{2}, \zeta_{1}$ and $\zeta_{2}$, we may find one and only one case where this particular set of inputs belongs to. As $h_{1,2}$ and $\zeta_{1,2}$ are functions of $x$, which case the inputs will fall into is position dependent.

Let us use an example to illustrate how Table 1 can be used to solve for $K$. Suppose the inputs are as follows: $n=0.5, q=1, \zeta_{1}=1, \zeta_{2}=0.5$, and $h_{1}=h_{2}=1$. First, we may determine from the fifth column of the table that, because $\zeta_{1}-\zeta_{2}>0$, only cases $1(\mathrm{a}), 2$, 4(a), 6 and 7 are possible. Second, on evaluating $q /\left(h_{1}+h_{2}\right)=0.5$ and $\omega_{1} \zeta_{1}+\omega_{2} \zeta_{2}=0.625$, 
Table 1: Seven possible cases are defined according to the values of $K$ and $z \equiv \tau_{c} / K$. In the last column, $\omega_{1}=n /(1+2 n)$ and $\omega_{2}=(1+n) /(1+2 n)$.

\begin{tabular}{|c|c|c|c|c|c|}
\hline Case & $K$ & $z$ & $\tau=-K(y-z)$ & $\zeta_{1}-\zeta_{2}$ & $q /\left(h_{1}+h_{2}\right)$ \\
\hline 1 & $>0$ & $\begin{array}{l}\geq-h_{2} \\
\leq h_{1}\end{array}$ & $\begin{array}{ll}>0 & \text { if }-h_{2} \leq y<z \\
=0 & \text { if } y=z \\
<0 & \text { if } z<y \leq h_{1}\end{array}$ & $\begin{array}{l}\text { (a) }>0 \Leftrightarrow \frac{h_{1}-h_{2}}{2}<z \leq h_{1} \\
\text { (b) }=0 \Leftrightarrow z=\frac{h_{1}-h_{2}}{2} \\
\text { (c) }<0 \Leftrightarrow-h_{2} \leq z<\frac{h_{1}-h_{2}}{2}\end{array}$ & $\begin{array}{l}\text { (a) } \geq \omega_{2} \zeta_{1}+\omega_{1} \zeta_{2} \\
\text { (b) }>\zeta_{2} \\
\text { (c) } \geq \omega_{1} \zeta_{1}+\omega_{2} \zeta_{2}\end{array}$ \\
\hline 2 & $>0$ & $>h_{1}$ & $>0$ & $>0$ & $\begin{array}{l}>\frac{1}{2}\left(\zeta_{1}+\zeta_{2}\right) \\
<\omega_{2} \zeta_{1}+\omega_{1} \zeta_{2}\end{array}$ \\
\hline 3 & $>0$ & $<-h_{2}$ & $<0$ & $<0$ & $\begin{array}{l}>\frac{1}{2}\left(\zeta_{1}+\zeta_{2}\right) \\
<\omega_{1} \zeta_{1}+\omega_{2} \zeta_{2}\end{array}$ \\
\hline 4 & $<0$ & $\begin{array}{l}\geq-h_{2} \\
\leq h_{1}\end{array}$ & $\begin{array}{ll}<0 & \text { if }-h_{2} \leq y<z \\
=0 & \text { if } y=z \\
>0 & \text { if } z<y \leq h_{1}\end{array}$ & $\begin{array}{l}\text { (a) }>0 \Leftrightarrow-h_{2} \leq z<\frac{h_{1}-h_{2}}{2} \\
\text { (b) }=0 \Leftrightarrow z=\frac{h_{1}-h_{2}}{2} \\
\text { (c) }<0 \Leftrightarrow \frac{h_{1}-h_{2}}{2}<z \leq h_{1}\end{array}$ & $\begin{array}{l}\text { (a) } \leq \omega_{1} \zeta_{1}+\omega_{2} \zeta_{2} \\
\text { (b) }<\zeta_{2} \\
\text { (c) } \leq \omega_{2} \zeta_{1}+\omega_{1} \zeta_{2}\end{array}$ \\
\hline 5 & $<0$ & $>h_{1}$ & $<0$ & $<0$ & $\begin{array}{l}>\omega_{2} \zeta_{1}+\omega_{1} \zeta_{2} \\
<\frac{1}{2}\left(\zeta_{1}+\zeta_{2}\right)\end{array}$ \\
\hline 6 & $<0$ & $<-h_{2}$ & $>0$ & $>0$ & $\begin{array}{l}>\omega_{1} \zeta_{1}+\omega_{2} \zeta_{2} \\
<\frac{1}{2}\left(\zeta_{1}+\zeta_{2}\right)\end{array}$ \\
\hline 7 & $=0$ & $\mathrm{~N} / \mathrm{A}$ & $\mathrm{N} / \mathrm{A}$ & Any value & $=\frac{1}{2}\left(\zeta_{1}+\zeta_{2}\right)$ \\
\hline
\end{tabular}

we may further determine from the sixth column that this set of inputs should fall into case 4(a). Then, we may proceed to solve Eq. (A 3) for $z$, where $-h_{2} \leq z<\left(h_{1}-h_{2}\right) / 2$, and the functions $G$ and $F$ are given by Eqs. (A 18) and (A 19). Equation (A 3) can be solved numerically, using Matlab or other similar packages, to generate a unique root of the equation within the specified range. When $z$ is found, we then substitute it into Eq. (A 1$)$ to get $K$.

We have tested the accuracy of the numerical method by comparing results with those generated by the analytical or simpler solution methods for the special cases described in Sections 3.1-3.3. In the interest of space, the comparison of results is, however, not presented here. 


\section{Discussion}

Let us consider the following sinusoidal functions of $x$ for the axial distributions of the wall topographies and the zeta potentials:

$$
\begin{aligned}
& h_{1}(x)=1+h_{1}^{\prime} \cos (2 \pi x), \\
& h_{2}(x)=1+h_{2}^{\prime} \cos (2 \pi x+\phi), \\
& \zeta_{1}(x)=\bar{\zeta}_{1}+\zeta_{1}^{\prime} \cos \left(2 \pi x+\theta_{1}\right), \\
& \zeta_{2}(x)=\bar{\zeta}_{2}+\zeta_{2}^{\prime} \cos \left(2 \pi x+\theta_{2}\right),
\end{aligned}
$$

where $h_{1,2}^{\prime}$ and $\zeta_{1,2}^{\prime}$ are the amplitudes of the wall undulation and potential modulation on the upper/lower walls, $\bar{\zeta}_{1,2}$ are the average potentials on the two walls, and $\phi$ and $\theta_{1,2}$ are the phase shifts for the corresponding distributions.

\subsection{Flat and uniformly charged walls, but with unequal potentials}

We first look into the issue of linear superposition of the hydrodynamic and electrokinetic effects by considering flow through a channel with asymmetrically charged walls. For simplicity, we let the walls be flat $\left(h_{1,2}^{\prime}=0\right)$ and uniformly charged $\left(\zeta_{1,2}^{\prime}=0\right)$, but $\zeta_{1}$ can be different from $\zeta_{2}$.

We show in Fig. 2 the following types of flow rate as functions of $\zeta_{2}$ :

$$
\begin{gathered}
q_{\mathrm{PO}}=\text { rate of flow due to applied pressure gradient }(\Delta P=1) \text { only; } \\
q_{\mathrm{EO}}=\text { rate of flow due to electric forcing }\left(\zeta_{1}=1,0 \leq \zeta_{2} \leq 2\right) \text { only; } \\
q_{\mathrm{comb}}=\text { rate of flow due to the combined action of } \Delta P=1, \zeta_{1}=1,0 \leq \zeta_{2} \leq 2,
\end{gathered}
$$

where $q_{\mathrm{PO}}$ and $q_{\mathrm{EO}}$ can be computed readily by Eqs. (23) and (20), respectively, and $q_{\mathrm{comb}}$ has to be found numerically (except when $n=1$, for which Eq. (32) can be used, or when $\zeta_{1}=\zeta_{2}$, for which Eq. (23) can be used). It is clear from the figure that, for a non-Newtonian fluid $(n \neq 1)$, the sum of $q_{\mathrm{PO}}$ and $q_{\mathrm{EO}}$ (represented by the dashed lines) is in general not equal to $q_{\text {comb }}$. They are equal to each other only when $\zeta_{1}=\zeta_{2}$, corresponding to a symmetrical flow configuration. For a Newtonian fluid $(n=1)$, the sum of the flow rates due separately to 
the two forcings is always equal to the flow rate due to the simultaneous action of the two forcings, which is expected. Such linearity will not apply to a non-Newtonian fluid, except when the zeta potential is the same on the two walls, or when the flow is symmetrical about the centerline of the channel. The results here reveal that, even for flat and uniformly charged walls, a lack of symmetry about the channel centerline alone will upset the principle of linear superposition of the hydrodynamic and electric forcings for a non-Newtonian fluid. This can be reasoned as follows. Pure EOF in this case has a linear velocity profile, and the stress is the constant $\tau_{c}$ given by Eq. (19). In the case of symmetry $\zeta_{1}=\zeta_{2}$, the pure EOF has a plug flow profile, and $\tau_{c}=0$. The vanishing of stress associated with electrokinetics will enable the decoupling of the two forces in driving the flow. In the case of asymmetry, or $\zeta_{1} \neq \zeta_{2}$, the stress associated with the electrokinetics is not zero, and will interact in a nonlinear manner with the stress associated with the hydrodynamic forcing when the fluid is non-Newtonian.

\subsection{Synchronized walls: $\phi=\pi$}

When $\phi=\pi$, for which $h_{2}(x)=1-h_{2}^{\prime} \cos (2 \pi x)$, the two walls are in a synchronized configuration, i.e., the topmost/lowest points of the upper wall being aligned respectively with those of the lower wall.

An analytical expression for the flow rate is obtainable from Eq. (18) for Newtonian fluid $(n=1)$ :

$$
\begin{aligned}
\left.q\right|_{n=1}= & \frac{4-\left(h_{1}^{\prime}-h_{2}^{\prime}\right)^{2}}{6\left[8+\left(h_{1}^{\prime}-h_{2}^{\prime}\right)^{2}\right]}\left\{\Delta P\left[4-\left(h_{1}^{\prime}-h_{2}^{\prime}\right)^{2}\right]^{\frac{3}{2}}+12\left(\bar{\zeta}_{1}+\bar{\zeta}_{2}\right)\right. \\
& \left.-6\left(h_{1}^{\prime}-h_{2}^{\prime}\right)\left(\zeta_{1}^{\prime} \cos \theta_{1}+\zeta_{2}^{\prime} \cos \theta_{2}\right)\right\} \quad \text { for } \phi=\pi .
\end{aligned}
$$

If it is further assumed that the amplitudes of the two wall undulations are the same, i.e., $h_{1}^{\prime}=h_{2}^{\prime}$, such that channel height becomes a constant, $h_{1}(x)+h_{2}(x)=2$, the flow rate above reduces to

$$
\left.q\right|_{n=1}=\frac{2 \Delta P}{3}+\bar{\zeta}_{1}+\bar{\zeta}_{2}
$$

which depends only on the mean wall potentials, but not on the spatial-varying components of the wall shapes and zeta potentials. 
We show in Fig. 3 the flow rate $q$ as a function of $\theta_{1}$, for $\bar{\zeta}_{1,2}=\zeta_{1,2}^{\prime}=1, h_{1,2}^{\prime}=0.5$ and $\theta_{2}=\pi$. As expected, for $n=1, q$ is unaffected by the phase of $\zeta_{1}$, whether $\Delta P=0$ or not. It is interesting to find that, for $n \neq 1, q$ is also independent of $\theta_{1}$, but only when $\Delta P=0$. When $\Delta P \neq 0, q$ will have disparate dependence on $\theta_{1}$, depending on $n$. For $\Delta P=1$, as clearly shown in the figure, $q$ reaches the minimum and maximum when $\theta_{1}=0, \pi$, respectively, for $n=0.5$ (i.e., shear thinning). The opposite is true: maximum and minimum $q$ at $\theta_{1}=0, \pi$, respectively, for $n=2$ (i.e., shear thickening). We may infer from the results that, in the absence of applied pressure forcing, there is always no net effect due to the interaction between the potential modulation and the wall undulation on the flow through a sinuous channel of uniform depth. This statement applies to fluid of any power-law index $n$. When there is an applied pressure gradient, the nonlinear interaction between the pressure forcing and the electric forcing will lead to qualitatively different behaviors exhibited by shear-thinning and shear-thickening fluids. Under a favorable applied pressure gradient, a shear-thinning fluid $(n<1)$ will have the flow rate to reach the maximum/minimum when the two potential distributions are in-phase/ $\pi$-out-of-phase with each other, respectively. The reverse is true for a shear-thickening fluid $(n>1)$.

We further show in Fig. 4 other qualitatively different behaviors exhibited by shearthinning and shear-thickening fluids. In this figure, we may see how the flow rate is affected by the amplitude of the potential modulation, where $\zeta_{1}^{\prime}=\zeta_{2}^{\prime}=\zeta^{\prime}$, for $n=0.5,1,2$, and $\theta_{1}=0, \pi$. It is remarkable that, under a favorable applied pressure gradient $\Delta P>0$, the potential modulation $\zeta^{\prime}$ will have a positive effect on $q$ for $n<1$, but a negative effect on $q$ for $n>1$, where the effects, positive or negative, are the strongest at $\theta_{1}=\pi$. Under an adverse applied pressure gradient $\Delta P<0$, the effect of $\zeta^{\prime}$ on $q$ is reversed: negative for $n<1$, but positive for $n>1$.

\subsection{Flat uncharged lower wall}

We have seen from Fig. 3 that, for flow through a channel of uniform cross section, $q$ is independent of $n$ when $\Delta P=0$. This is no longer true for flow through a channel of varying cross section. In Fig. 5, we show the results for a channel with a lower wall that is flat 
$\left(h_{2}^{\prime}=0\right)$ and uncharged $\left(\zeta_{2}=0\right)$. Here, even for $\Delta P=0$, the flow rate changes as the phase of $\zeta_{1}$ varies. For any $n$, the flow rate is the minimum when $\theta_{1}=0$, and is the maximum when $\theta_{1}=\pi$. These two phases correspond, respectively, to the cases where the minimum or maximum $\zeta_{1}$ is positioned at the narrowest section of the channel. This is consistent with the previous finding [5] that it is the electric forcing at the narrowest part of the channel that determines the net flow. An analytical evidence is available from Eq. (31) for the Newtonian fluid flow. From this equation, we see that the wall undulation interacts with the potential modulation through the term $-6 h_{1}^{\prime} \zeta_{1}^{\prime} \cos \theta_{1}$, which is the maximum negative when $\theta_{1}=0$, and is the maximum positive when $\theta_{1}=\pi$, thereby giving the minimum and maximum flow rates at these phases, respectively.

\subsection{Symmetrical walls}

In the case shown above, which is for flow through an asymmetrical channel, the flow rate is the maximum for any $n$ when $\theta_{1}=\pi$. Let us now show a different case to illustrate that the optimum phase does not always occur exactly at $\theta_{1}=\pi$. We show in Fig. $6 q$ as a function of $\theta_{1}$, where $\zeta_{1}=\zeta_{2}=1+\cos \left(2 \pi x+\theta_{1}\right), h_{1}=h_{2}=1+0.5 \cos (2 \pi x)$, and $\Delta P=0$. In this case of symmetrical flow, the maximum flow rate occurs at $\theta_{1}=\pi$ only for $n \leq 1$, but not for $n>1$. For $n=2, q$ reaches the maximum at $\theta_{1} \approx 0.9 \pi$ and $1.1 \pi$. These are the phases at which the maximum wall potential is located slightly away from the narrowest section. Furthermore, in the case shown above in Fig. 5, $q$ increases with increasing $n$ monotonically at any $\theta_{1}$. The case shown here in Fig. 6 displays different trends: $q$ may increase or decrease with increasing $n$, depending on $\theta_{1}$. Near the two limits, $\theta_{1}=0$ and $\theta_{1}=\pi, q$ increases as $n$ decreases. For an intermediate $\theta$, the contrary is true.

We show in Fig. 7 the maximum flow rate $q_{\max }$ and the corresponding optimum phase $\theta_{1 \text { optm }}$ as functions of the power-law index $n$. Here, it is seen that $q_{\max }$ follows a non-monotonic relationship with $n$. It has a minimum value of approximately 1.99 at $n=1.27$. The optimum phase $\theta_{1 \mathrm{optm}}$, which is the phase at which the maximum flow rate occurs, is equal to $\pi$ for $n \leq 1$. It drops sharply from $\pi$ to approximately $0.9 \pi$, as $n$ increases above 1 . 


\section{Concluding remarks}

We have set out to investigate electroosmotic flow of a power-law fluid through a slit channel bounded by asymmetric walls with periodic variations in shape and zeta potential. The problem we have studied is simplified by the lubrication approximation, and the use of the electroosmotic slip boundary condition on assuming that the bulk fluid is shielded from the wall by a very thin Newtonian depletion layer enclosing completely the electric double layer. Without the mid-plane symmetry condition, the problem becomes more difficult to solve. By identifying all the possible relations between the flow rate and the given distributions of zeta potential and wall shape, we have devised a numerical scheme to determine the axial distribution of the pressure gradient.

Our results have revealed that even when the walls are flat and uniformly charged, a lack of mid-plane symmetry alone will invalidate the principle of linear superposition of forces found by Berli and Olivares [2] for electrokinetic flow of a non-Newtonian fluid in a channel with the depletion effects taken into account. We have also found that, by varying the phase shift between the patterns on the two walls, dramatically different behaviors will be exhibited by shear-thinning and shear-thickening fluids. How the wall undulation may interact with the potential modulation is strongly dependent on their phase difference, the power-law behavior index, and the applied pressure gradient. We have demonstrated in several examples how contrasting flow phenomena may arise under different values of these parameters. For instance, for flow in a channel with synchronized walls forced by a favorable pressure gradient, the flow rate will reach the maximum/minimum for a shear-thinning fluid, but the minimum/maximum for a shear-thickening fluid, when the zeta potential distributions on the two walls are in-phase/ $\pi$-out-of-phase with each other, respectively. The contrary is true (i.e., the maximum becomes the minimum, and vice versa) when the pressure gradient is unfavorable. These effects may have important implications in the electrokinetic transport of complex fluids in a microchannel with surface heterogeneities. It is desirable if the findings of the present study can be verified by experiments in the future. 


\section{Acknowledgments}

Comments by the two anonymous reviewers are gratefully acknowledged. The work was supported by the Research Grants Council of the Hong Kong Special Administrative Region, China, through Project No. HKU 715510E, and also by the University of Hong Kong through the Small Project Funding Scheme under Project Code 201309176109. 


\section{Appendix}

Introducing $z \equiv \tau_{c} / K$, we may rewrite Eqs. (11) and (12) in the following forms:

$$
\frac{n}{1+n}|K|^{\frac{1}{n}} G(z)=\zeta_{1}-\zeta_{2}
$$

and

$$
q=\zeta_{2}\left(h_{1}+h_{2}\right)+\frac{n}{1+n}|K|^{\frac{1}{n}} F(z),
$$

where the two functions $G(z)$ and $F(z)$ stem from the integrals in Eqs. (11) and (12). For individual cases, these functions can be expressed analytically, as presented below. Eliminating $K$ from Eqs. (A 1$)$ and (A 2) gives

$$
q=\zeta_{2}\left(h_{1}+h_{2}\right)+\left(\zeta_{1}-\zeta_{2}\right)[F / G] \quad \text { if } G \neq 0 .
$$

When $G \neq 0$, the equation above will be solved numerically for $z$, for any given $q, h_{1,2}, \zeta_{1,2}$, and $n$. A more direct way to find $z$ is used when $G=0$, which will happen only in cases 1 and 4. Putting $z$ back to Eq. (A 1), we may determine $K$.

\section{A.1 Case 1}

For case $1\left(K>0,-h_{2} \leq z \leq h_{1}\right)$, the functions $G$ and $F$ are

$$
\begin{gathered}
G=\left(z+h_{2}\right)^{1+\frac{1}{n}}-\left(h_{1}-z\right)^{1+\frac{1}{n}} \\
F=\frac{1+n}{1+2 n}\left(z+h_{2}\right)^{2+\frac{1}{n}}+\left(h_{1}-z\right)\left(z+h_{2}\right)^{1+\frac{1}{n}}-\frac{n}{1+2 n}\left(h_{1}-z\right)^{2+\frac{1}{n}} .
\end{gathered}
$$

By virtue of the monotonicity of $G$, one may infer from Eq. (A 1) that

$$
\zeta_{1}-\zeta_{2}\left\{\begin{aligned}
>0 & \Leftrightarrow\left(h_{1}-h_{2}\right) / 2<z \leq h_{1}, \\
=0 & \Leftrightarrow z=\left(h_{1}-h_{2}\right) / 2, \\
<0 & \Leftrightarrow-h_{2} \leq z<\left(h_{1}-h_{2}\right) / 2 .
\end{aligned}\right.
$$

When $\zeta_{1}-\zeta_{2}=0$, corresponding to $G=0$, Eq. (A 3) is inapplicable. Instead, we may directly substitute $z=\left(h_{1}-h_{2}\right) / 2$ into Eq. (A 2) and get

$$
K=\left(1+\frac{1}{2 n}\right)^{n}\left(\frac{h_{1}+h_{2}}{2}\right)^{-1-2 n} \times\left[q-\zeta_{2}\left(h_{1}+h_{2}\right)\right]^{n} \quad \text { for } \zeta_{1}-\zeta_{2}=0 .
$$


In this particular case, $K$ is solvable if and only if $q$ is larger than $\zeta_{2}\left(h_{1}+h_{2}\right)$.

When $\zeta_{1}-\zeta_{2} \neq 0$, corresponding to $G \neq 0, F / G$ is a monotonically decreasing function of z. Also,

$$
F / G\left\{\begin{array}{l}
\geq \omega_{2}\left(h_{1}+h_{2}\right) \quad \text { iff }\left(h_{1}-h_{2}\right) / 2<z \leq h_{1}, \\
\leq \omega_{1}\left(h_{1}+h_{2}\right) \quad \text { iff }-h_{2} \leq z<\left(h_{1}-h_{2}\right) / 2,
\end{array}\right.
$$

where $\omega_{1}=n /(1+2 n)$ and $\omega_{2}=(1+n) /(1+2 n)$. It follows from Eqs. (A 3), (A 6) and (A 8) that

$$
q \geq \begin{cases}\left(\omega_{2} \zeta_{1}+\omega_{1} \zeta_{2}\right)\left(h_{1}+h_{2}\right) & \text { for } \zeta_{1}-\zeta_{2}>0 \text { or }\left(h_{1}-h_{2}\right) / 2<z \leq h_{1} \\ \left(\omega_{1} \zeta_{1}+\omega_{2} \zeta_{2}\right)\left(h_{1}+h_{2}\right) & \text { for } \zeta_{1}-\zeta_{2}<0 \text { or }-h_{2} \leq z<\left(h_{1}-h_{2}\right) / 2\end{cases}
$$

\section{A.2 Case 2}

For case $2\left(K>0, z>h_{1}\right)$, the functions $G$ and $F$ are

$$
\begin{gathered}
G=-\left(z-h_{1}\right)^{1+\frac{1}{n}}+\left(z+h_{2}\right)^{1+\frac{1}{n}} \\
F=\frac{n}{1+2 n}\left(z-h_{1}\right)^{2+\frac{1}{n}}+\left(h_{1}+h_{2}\right)\left(z+h_{2}\right)^{1+\frac{1}{n}}-\frac{n}{1+2 n}\left(z+h_{2}\right)^{2+\frac{1}{n}} .
\end{gathered}
$$

Again, $G$ is an increasing function of $z$, and hence $G \geq\left(h_{1}+h_{2}\right)^{1+1 / n}>0$. It follows from Eq. (A 1$)$ that $\zeta_{1}-\zeta_{2}>0$. Given that $F / G$ is a decreasing function of $z$, the upper and lower bounds for $q$ can be obtained as follows:

$$
\begin{aligned}
q_{\max } & \rightarrow \zeta_{2}\left(h_{1}+h_{2}\right)+\left(\zeta_{1}-\zeta_{2}\right) \times\left.\frac{F}{G}\right|_{z=h_{1}}=\left(\omega_{2} \zeta_{1}+\omega_{1} \zeta_{2}\right)\left(h_{1}+h_{2}\right), \\
q_{\min } & \rightarrow \zeta_{2}\left(h_{1}+h_{2}\right)+\left(\zeta_{1}-\zeta_{2}\right) \times\left.\frac{F}{G}\right|_{z \rightarrow+\infty}=\frac{1}{2}\left(\zeta_{1}+\zeta_{2}\right)\left(h_{1}+h_{2}\right) .
\end{aligned}
$$

\section{A.3 Case 3}

For case $3\left(K>0, z<-h_{2}\right)$, the functions $G$ and $F$ are

$$
\begin{gathered}
G=-\left(-z+h_{1}\right)^{1+\frac{1}{n}}+\left(-z-h_{2}\right)^{1+\frac{1}{n}} \\
F=\frac{n}{1+2 n}\left(-z-h_{2}\right)^{2+\frac{1}{n}}+\left(h_{1}+h_{2}\right)\left(-z-h_{2}\right)^{1+\frac{1}{n}}-\frac{n}{1+2 n}\left(-z+h_{1}\right)^{2+\frac{1}{n}} .
\end{gathered}
$$


The function $G$ is always negative in this case, and therefore $\zeta_{1}-\zeta_{2}<0$ by Eq. (A 1$)$. Again, $F / G$ is a decreasing function of $z$. Hence, the range of $q$ is

$$
\begin{gathered}
q_{\max } \rightarrow \zeta_{2}\left(h_{1}+h_{2}\right)+\left(\zeta_{1}-\zeta_{2}\right) \times\left.\frac{F}{G}\right|_{z \rightarrow-h_{2}}=\left(\omega_{1} \zeta_{1}+\omega_{2} \zeta_{2}\right)\left(h_{1}+h_{2}\right), \\
q_{\min } \rightarrow \zeta_{2}\left(h_{1}+h_{2}\right)+\left(\zeta_{1}-\zeta_{2}\right) \times\left.\frac{F}{G}\right|_{z \rightarrow-\infty}=\frac{1}{2}\left(\zeta_{1}+\zeta_{2}\right)\left(h_{1}+h_{2}\right) .
\end{gathered}
$$

\section{A.4 Case 4}

For case $4\left(K<0,-h_{2} \leq z \leq h_{1}\right)$, the functions $G$ and $F$ are

$$
\begin{gathered}
G=\left(h_{1}-z\right)^{1+\frac{1}{n}}-\left(z+h_{2}\right)^{1+\frac{1}{n}} \\
F=-\frac{1+n}{1+2 n}\left(z+h_{2}\right)^{2+\frac{1}{n}}-\left(h_{1}-z\right)\left(z+h_{2}\right)^{1+\frac{1}{n}}+\frac{n}{1+2 n}\left(h_{1}-z\right)^{2+\frac{1}{n}} .
\end{gathered}
$$

The deduction here is similar to that presented above for case 1. By virtue of the monotonicity of $G$, one may infer from Eq. (A 1) that

$$
\zeta_{1}-\zeta_{2}\left\{\begin{aligned}
>0 & \Leftrightarrow \quad-h_{2} \leq z<\left(h_{1}-h_{2}\right) / 2, \\
=0 & \Leftrightarrow z=\left(h_{1}-h_{2}\right) / 2, \\
<0 & \Leftrightarrow \quad\left(h_{1}-h_{2}\right) / 2<z \leq h_{1} .
\end{aligned}\right.
$$

When $\zeta_{1}-\zeta_{2}=0$, we may substitute $z=\left(h_{1}-h_{2}\right) / 2$ into Eq. (A 2$)$ to get

$$
K=-\left(1+\frac{1}{2 n}\right)^{n}\left(\frac{h_{1}+h_{2}}{2}\right)^{-1-2 n} \times\left[\zeta_{2}\left(h_{1}+h_{2}\right)-q\right]^{n} \quad \text { for } \zeta_{1}-\zeta_{2}=0 .
$$

In this particular case, $K$ is solvable if and only if $q$ is smaller than $\zeta_{2}\left(h_{1}+h_{2}\right)$.

When $\zeta_{1}-\zeta_{2} \neq 0, F / G$ is a decreasing function of $z$, satisfying

$$
F / G \begin{cases}\leq \omega_{1}\left(h_{1}+h_{2}\right) & \text { iff }-h_{2} \leq z<\left(h_{1}-h_{2}\right) / 2 \\ \geq \omega_{2}\left(h_{1}+h_{2}\right) & \text { iff }\left(h_{1}-h_{2}\right) / 2<z \leq h_{1}\end{cases}
$$

The ranges for $q$ can be found accordingly via Eq. (A 3):

$$
q \begin{cases}\geq\left(\omega_{1} \zeta_{1}+\omega_{2} \zeta_{2}\right)\left(h_{1}+h_{2}\right) & \text { for } \zeta_{1}-\zeta_{2}>0, \text { or }-h_{2} \leq z<\left(h_{1}-h_{2}\right) / 2 \\ \geq\left(\omega_{2} \zeta_{1}+\omega_{1} \zeta_{2}\right)\left(h_{1}+h_{2}\right) & \text { for } \zeta_{1}-\zeta_{2}<0, \text { or }\left(h_{1}-h_{2}\right) / 2<z \leq h_{1}\end{cases}
$$




\section{A.5 Case 5}

For case $5\left(K<0, z>h_{1}\right)$, the functions $G$ and $F$ are

$$
\begin{gathered}
G=\left(z-h_{1}\right)^{1+\frac{1}{n}}-\left(z+h_{2}\right)^{1+\frac{1}{n}} ; \\
F=\frac{n}{1+2 n}\left(z+h_{2}\right)^{2+\frac{1}{n}}-\left(h_{1}+h_{2}\right)\left(z+h_{2}\right)^{1+\frac{1}{n}}-\frac{n}{1+2 n}\left(z-h_{1}\right)^{2+\frac{1}{n}} .
\end{gathered}
$$

The function $G$ is always negative in this case, and hence by Eq. (A 1$), \zeta_{1}-\zeta_{2}<0$. The upper and lower bounds for $q$ in this case can be obtained as follows:

$$
\begin{gathered}
q_{\max } \rightarrow \zeta_{2}\left(h_{1}+h_{2}\right)+\left(\zeta_{1}-\zeta_{2}\right) \times\left.\frac{F}{G}\right|_{z \rightarrow+\infty}=\frac{1}{2}\left(\zeta_{1}+\zeta_{2}\right)\left(h_{1}+h_{2}\right), \\
q_{\min } \rightarrow \zeta_{2}\left(h_{1}+h_{2}\right)+\left(\zeta_{1}-\zeta_{2}\right) \times\left.\frac{F}{G}\right|_{z \rightarrow h_{1}}=\left(\omega_{2} \zeta_{1}+\omega_{1} \zeta_{2}\right)\left(h_{1}+h_{2}\right) .
\end{gathered}
$$

\section{A.6 Case 6}

For case $6\left(K<0, z<-h_{2}\right)$, the functions $G$ and $F$ are

$$
\begin{gathered}
G=\left(-z+h_{1}\right)^{1+\frac{1}{n}}-\left(-z-h_{2}\right)^{1+\frac{1}{n}} \\
F=\frac{n}{1+2 n}\left(-z+h_{1}\right)^{2+\frac{1}{n}}-\left(h_{1}+h_{2}\right)\left(-z-h_{2}\right)^{1+\frac{1}{n}}-\frac{n}{1+2 n}\left(-z-h_{2}\right)^{2+\frac{1}{n}}
\end{gathered}
$$

The function $G$ is always positive in this case, which by Eq, (A 1$)$ implies that $\zeta_{1}-\zeta_{2}>0$. The upper and lower bounds for $q$ in this case can be obtained as follows:

$$
\begin{gathered}
q_{\max } \rightarrow \zeta_{2}\left(h_{1}+h_{2}\right)+\left(\zeta_{1}-\zeta_{2}\right) \times\left.\frac{F}{G}\right|_{z \rightarrow-\infty}=\frac{1}{2}\left(\zeta_{1}+\zeta_{2}\right)\left(h_{1}+h_{2}\right), \\
q_{\min } \rightarrow \zeta_{2}\left(h_{1}+h_{2}\right)+\left(\zeta_{1}-\zeta_{2}\right) \times\left.\frac{F}{G}\right|_{z \rightarrow-h_{2}}=\left(\omega_{1} \zeta_{1}+\omega_{2} \zeta_{2}\right)\left(h_{1}+h_{2}\right) .
\end{gathered}
$$

\section{A. 7 Case 7}

For case $7(K=0)$, the shear stress reduces to $\tau=\tau_{c}$. The flow rate $q$ can be found readily from Eqs. (11) and (12) as follows:

$$
q=\frac{1}{2}\left(\zeta_{1}+\zeta_{2}\right)\left(h_{1}+h_{2}\right)
$$




\section{References}

[1] J.H. Masliyah, S. Bhattacharjee, Electrokinetic and Colloid Transport Phenomena, Wiley, New Jersey, 2006.

[2] C.L.A. Berli, M.L. Olivares, Electrokinetic flow of non-Newtonian fluids in microchannels, J. Colloid Interface Sci. 320 (2008) 582-589.

[3] H.A. Barnes, A review of the slip (wall depletion) of polymer solutions, emulsions and particle suspensions in viscometers: its cause, character, and cure, J. Non-Newtonian Fluid Mech. 56 (1995) 221-251.

[4] R. Tuinier, T. Taniguchi, Polymer depletion-induced slip near an interface, J. Phys.: Condens. Matter 17 (2005) L9-L14.

[5] C.O. Ng, C. Qi, Electroosmotic flow of a power-law fluid in a non-uniform microchannel, J. Non-Newtonian Fluid Mech. 208-209 (2014) 118-125.

[6] S. Das, S. Chakraborty, Analytical solutions for velocity, temperature and concentration distribution in electroosmotic microchannel flows of a non-Newtonian bio-fluid, Anal. Chim. Acta 559 (2006) 15-24.

[7] S. Chakraborty, Electroosmotically driven capillary transport of typical non-Newtonian biofluids in rectangular microchannels, Anal. Chim. Acta 605 (2007) 175-184.

[8] C. Zhao, E. Zholkovskij, J. Masliyah, C. Yang, Analysis of electroosmotic flow of powerlaw fluids in a slit microchannel, J. Colloid Interface Sci. 326 (2008) 503-510.

[9] R.P. Bharti, D.J.E. Harvie, M.R. Davidson, Electroviscous effects in steady fully developed flow of a power-law liquid through a cylindrical microchannel, Int. J. Heat Fluid Flow 30 (2009) 804-811.

[10] M.L. Olivares, L. Vera-Candioti, C.L.A. Berli, The EOF of polymer solutions, Electrophoresis 30 (2009) 921-929.

[11] G.H. Tang, X.F. Li, Y.L. He, W.Q. Tao, Electroosmotic flow of non-Newtonian fluid in microchannels, J. Non-Newtonian Fluid Mech. 157 (2009) 133-137. 
[12] C. Zhao, C. Yang, Nonlinear Smoluchowski velocity for electroosmosis of power-law fluids over a surface with arbitrary zeta potentials, Electrophoresis 31 (2010) 973-979.

[13] C.L.A. Berli, Output pressure and efficiency of electrokinetic pumping of nonNewtonian fluids, Microfluid. Nanofluid. 8 (2010) 197-207.

[14] N. Vasu, S. De, Electroviscous effects in purely pressure driven flow and stationary plane analysis in electroosmotic flow of power-law fluids in a slit microchannel, Int. J. Eng. Sci. 48 (2010) 1641-1658.

[15] N. Vasu, S. De, Electroosmotic flow of power-law fluids at high zeta potentials, Colloids and Surfaces A: Physicochem. Eng. Aspects 368 (2010) 44-52.

[16] A. Babaie, A. Sadeghi, M.H. Saidi, Combined electroosmotically and pressure driven flow of power-law fluids in a slit microchannel, J. Non-Newtonian Fluid Mech. 166 (2011) 792-798.

[17] A. Bandopadhyay, S. Chakraborty, Steric-effect induced alterations in streaming potential and energy transfer efficiency of non-Newtonian fluids in narrow confinements, Langmuir, 27 (2011) 12243-12252.

[18] M. Hadigol, R. Nosrati, M. Raisee, Numerical analysis of mixed electroosmotic/pressure driven flow of power-law fluids in microchannels and micropumps, Colloids and Surfaces A: Physicochem. Eng. Aspects 374 (2011) 142-153.

[19] A. Sadeghi, M. Fattahi, M.H. Saidi, An approximate analytical solution for electroosmotic flow of power-law fluids in a planar microchannel, J. Heat Trans. 133 (2011) 091701.

[20] C. Zhao, C. Yang, An exact solution for electroosmosis of non-Newtonian fluids in microchannels, J. Non-Newtonian Fluid Mech. 166 (2011) 1076-1079.

[21] C.C. Cho, C.L. Chen, C.K. Chen, Electrokinetically-driven non-Newtonian fluid flow in rough microchannel with complex-wavy surface, J. Non-Newtonian Fluid Mech. 173174 (2012) 13-20. 
[22] C.C. Cho, C.L. Chen, C.K. Chen, Flow characteristics and mixing performance of electrokinetically driven non-Newtonian fluid in contraction-expansion microchannel, Rheol. Acta 51 (2012) 925-935.

[23] S.Y. Deng, Y.J. Jian, Y.H. Bi, L. Chang, H.J. Wang, Q.S. Liu, Unsteady electroosmotic flow of power-law fluid in a rectangular microchannel, Mech. Res. Comm. 39 (2012) 914.

[24] M. Shamshiri, R. Khazaeli, M. Ashrafizaadeh, S. Mortazavi, Electroviscous and thermal effects on non-Newtonian liquid flows through microchannels, J. Non-Newtonian Fluid Mech. 173-174 (2012) 1-12.

[25] M.A. Vakili, A. Sadeghi, M.H. Saidi, A.A. Mozafari, Electrokinetically driven fluidic transport of power-law fluids in rectangular microchannels, Colloids and Surfaces A: Physicochem. Eng. Aspects 414 (2012) 440-456.

[26] C.O. Ng, C. Qi, Electroosmotic flow of a viscoplastic material through a slit channel with walls of arbitrary zeta potential, Phys. Fluids 25 (2013) 103102.

[27] C. Zhao, C. Yang, Electroosmotic flows of non-Newtonian power-law fluids in a cylindrical microchannel, Electrophoresis 34 (2013) 662-667.

[28] J. Dhar, U. Ghosh, S. Chakraborty, Alterations in streaming potential in presence of time periodic pressure-driven flow of a power law fluid in narrow confinements with nonelectrostatic ion-ion interactions, Electrophoresis 35 (2014) 662-669.

[29] Q. Zhu, S. Deng, Y. Chen, Periodical pressure-driven electrokinetic flow of power-law fluids through a rectangular microchannel, J. Non-Newtonian Fluid Mech. 203 (2014) $38-50$.

[30] A.D. Stroock, M. Weck, D.T. Chiu, W.T.S. Huck, P.J.A. Kenis, R.F. Ismagilov, G.M. Whitesides, Patterning electro-osmotic flow with patterned surface charge, Phys. Rev. Lett. 84.15 (2000) 3314.

[31] S.L.R. Barker, D. Ross, M.J. Tarlov, M. Gaitan, L.E. Locascio, Control of flow direction in microfluidic devices with polyelectrolyte multilayers, Anal. Chem. 72 (2000) 59255929. 
[32] W.L.W. Hau, D.W. Trau, N.J. Sucher, M. Wong, Y. Zohar, Surface-chemistry technology for microfluidics, J. Micromech. Microeng. 13 (2003) 272.

[33] E. Biddiss, D. Erickson, D. Li, Heterogeneous surface charge enhanced micromixing for electrokinetic flows, Anal. Chem. 76 (2004) 3208-3213.

[34] D. Yan, C. Yang, J. Miao, Y. Lam, X. Huang, Enhancement of electrokinetically driven microfluidic T-mixer using frequency modulated electric field and channel geometry effects, Electrophoresis 30 (2009) 3144-3152.

[35] W.M. Deen, Analysis of Transport Phenomena, second ed., Oxford University Press, New York, 2012.

[36] L.G. Leal, Advanced Transport Phenomena, Cambridge University Press, Cambridge, 2007.

[37] J.M. MacInnes, X. Du, R.W.K. Allen, Prediction of electrokinetic and pressure flow in microchannel T-junction, Phys. Fluids 15 (2003), 1992-2005.

[38] W.B. Zimmerman, J.M. Rees, T.J. Craven, Rheometry of non-Newtonian electrokinetic flow in a microchannel T-junction, Microfluid. Nanofluid. 2 (2006) 481-492.

[39] J.M. MacInnes, Computation of reacting electrokinetic flow in microchannel geometries, Chem. Eng. Sci. 57 (2002) 4539-4558. 


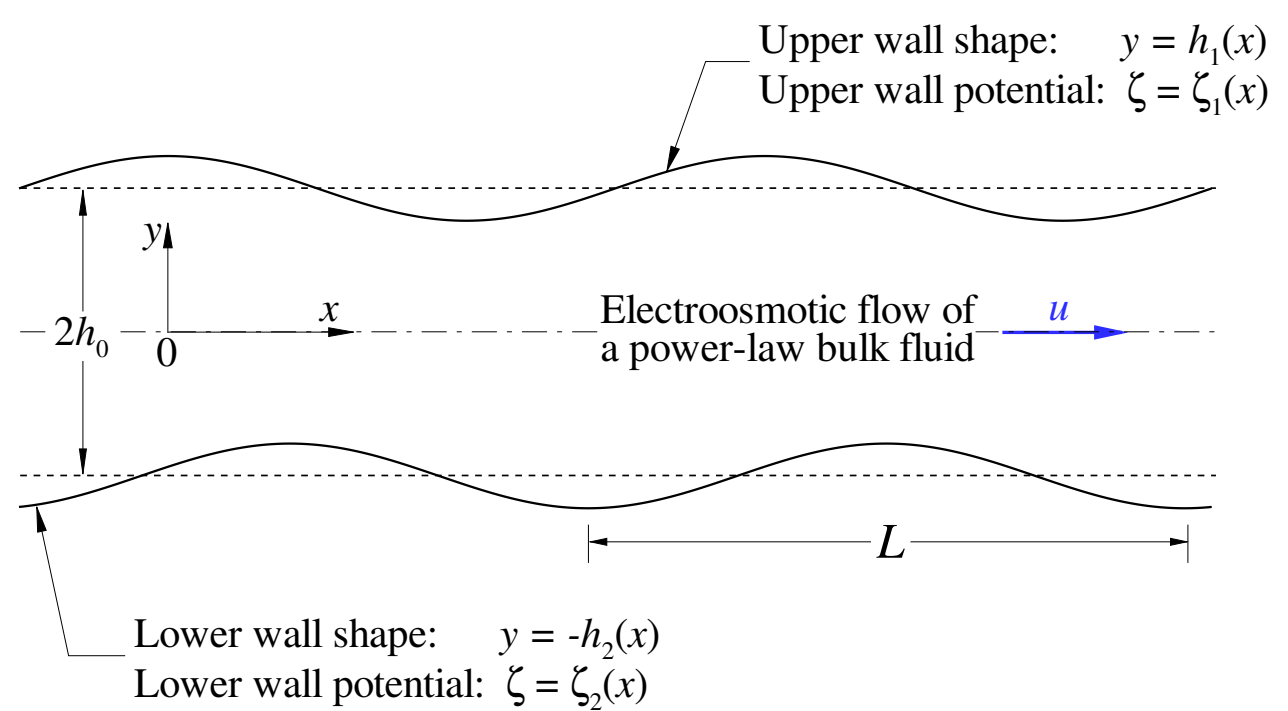

Figure 1: Combined pressure-driven and electroosmotic flow of a power-law bulk fluid through a slit channel with wall shapes and zeta potentials varying slowly and periodically in the axial direction, but not necessarily symmetrical about the centerline of the channel. 

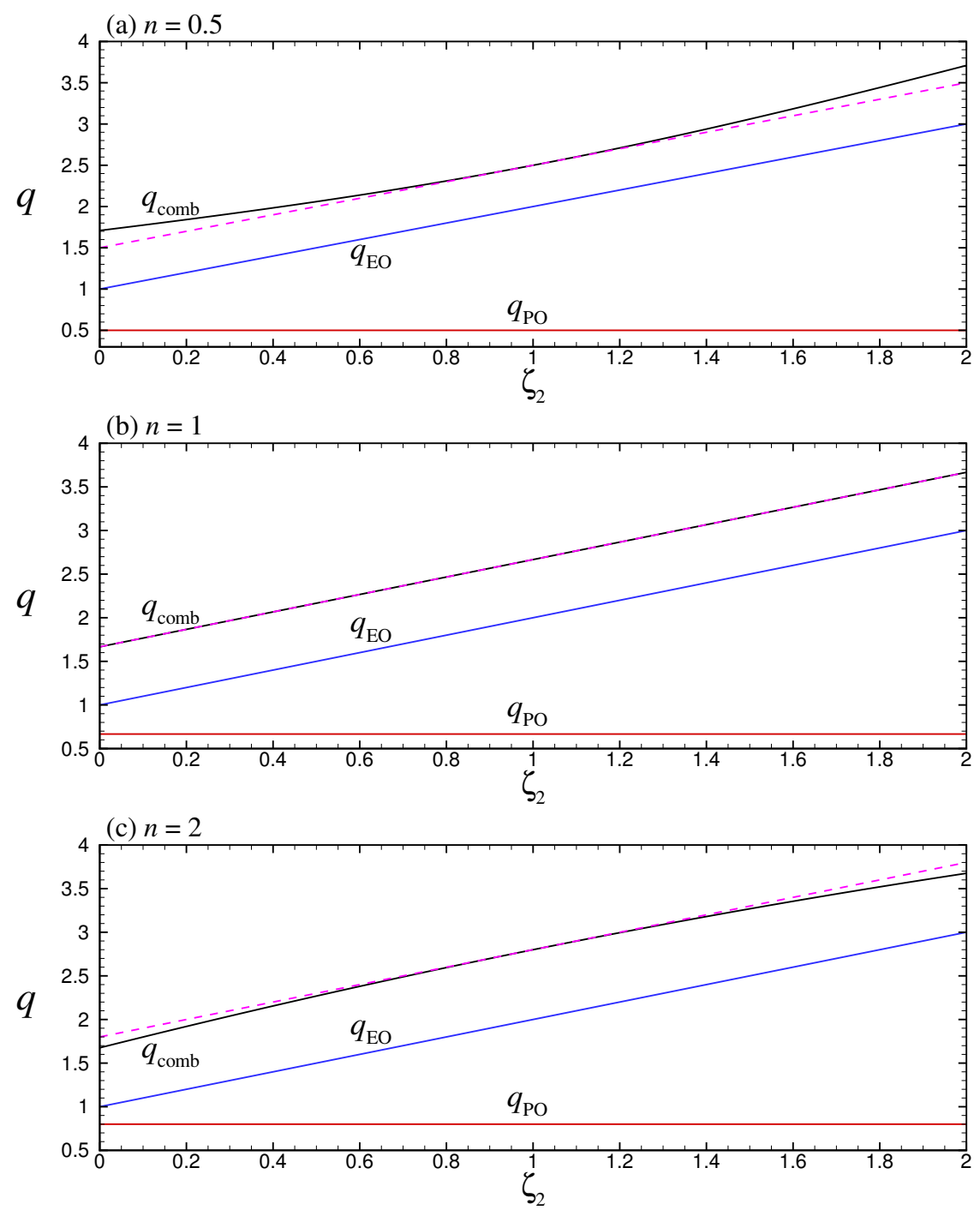

Figure 2: Flow rate $q$ as a function of the zeta potential on the lower wall $\zeta_{2}$ for $\Delta P=1$, $\zeta_{1}=1$, where the two walls are flat and uniformly charged, and (a) $n=0.5$, (b) $n=1$, (c) $n=2$, in which $q_{\mathrm{PO}}, q_{\mathrm{EO}}, q_{\mathrm{comb}}$ are the flow rates defined in Eqs. (28)-(30), and the dashed line represents $q_{\mathrm{PO}}+q_{\mathrm{EO}}$. For $n=1, q_{\mathrm{comb}}=q_{\mathrm{PO}}+q_{\mathrm{EO}}$. 

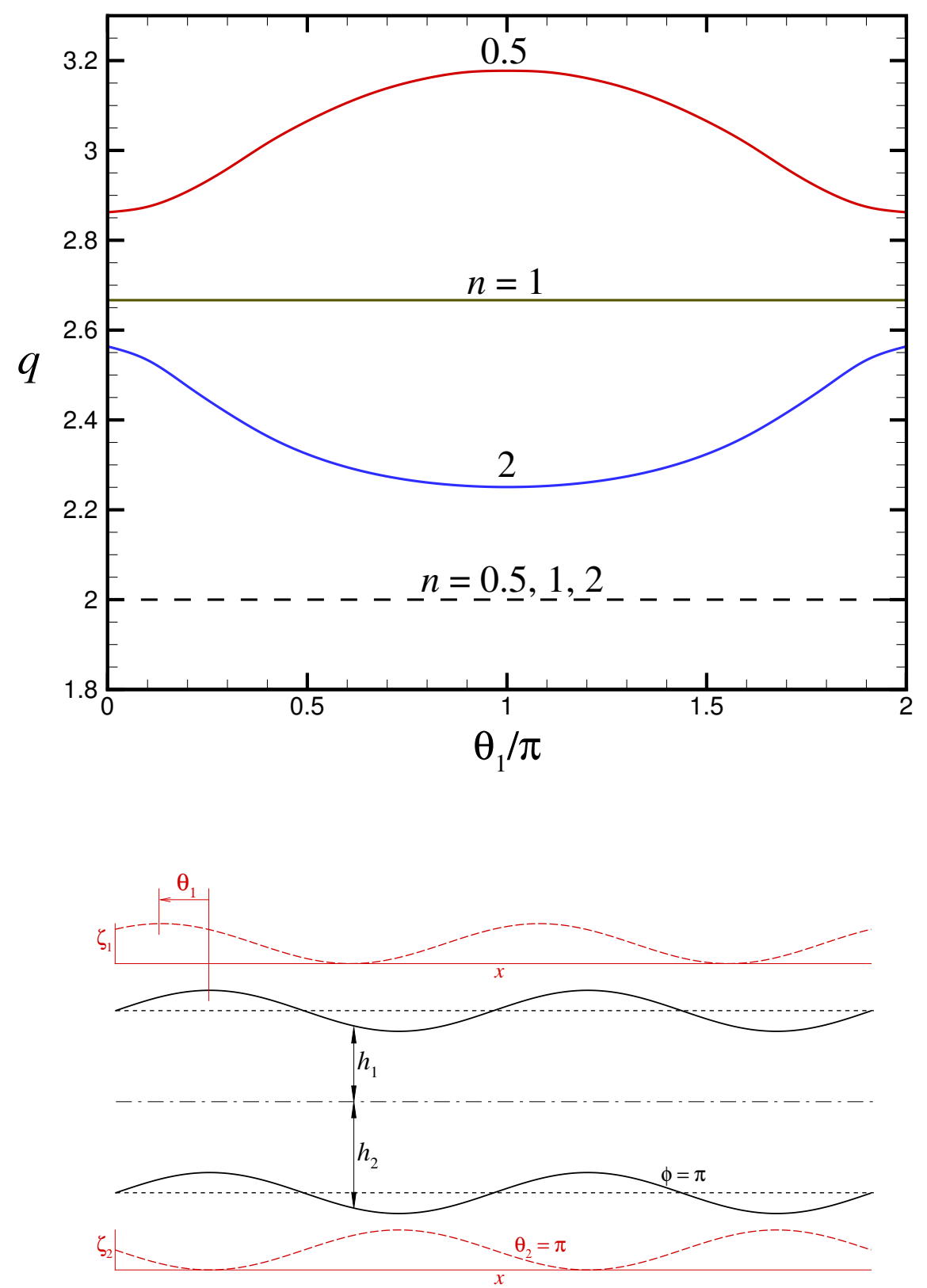

Figure 3: Flow rate $q$ as a function of the phase $\theta_{1}$, for $\Delta P=1$ (solid lines) and $\Delta P=0$ (dashed line), where the two walls are in a synchronized configuration: $\bar{\zeta}_{1}=\bar{\zeta}_{2}=1$, $\zeta_{1}^{\prime}=$ $\zeta_{2}^{\prime}=1, h_{1}^{\prime}=h_{2}^{\prime}=0.5, \phi=\pi$, and $\theta_{2}=\pi$. 
(a) $\Delta P=1, \theta_{1}=0$

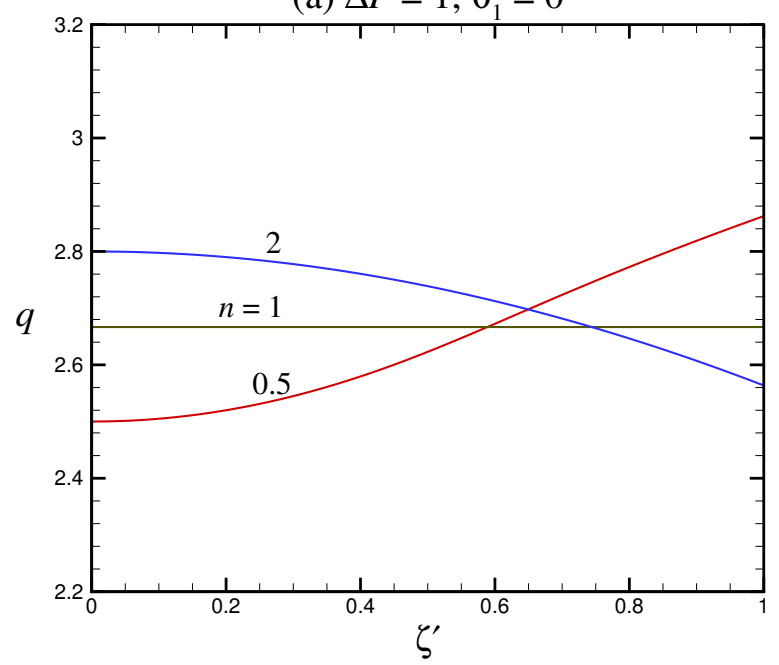

(c) $\Delta P=-1, \theta_{1}=0$

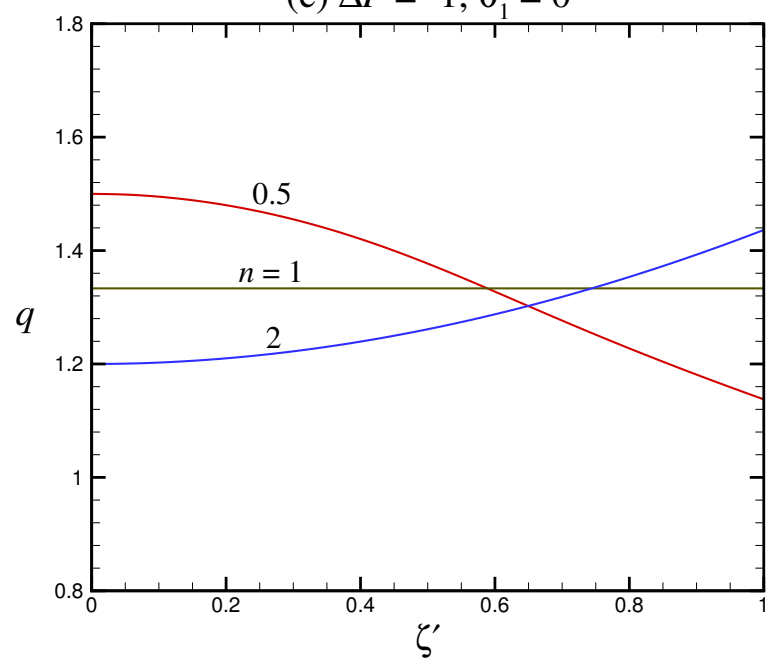

(b) $\Delta P=1, \theta_{1}=\pi$

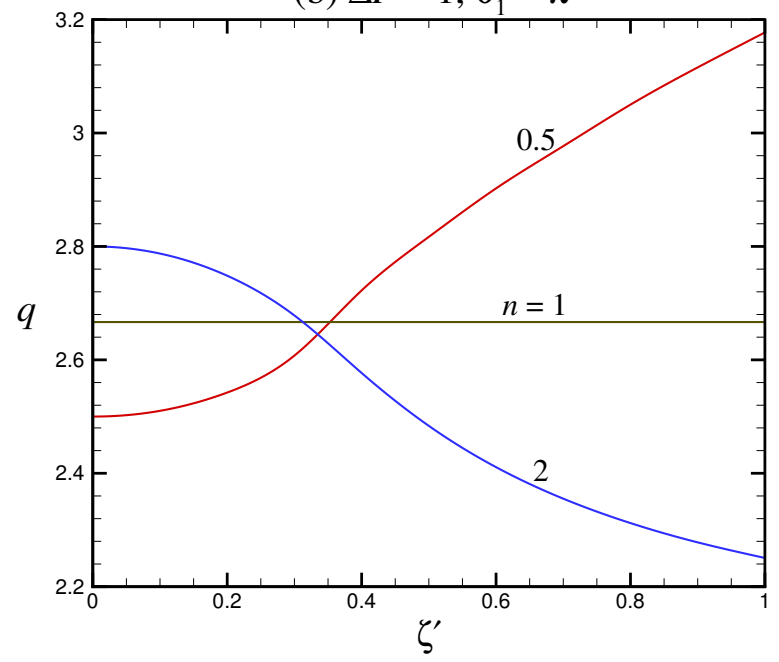

(d) $\Delta P=-1, \theta_{1}=\pi$

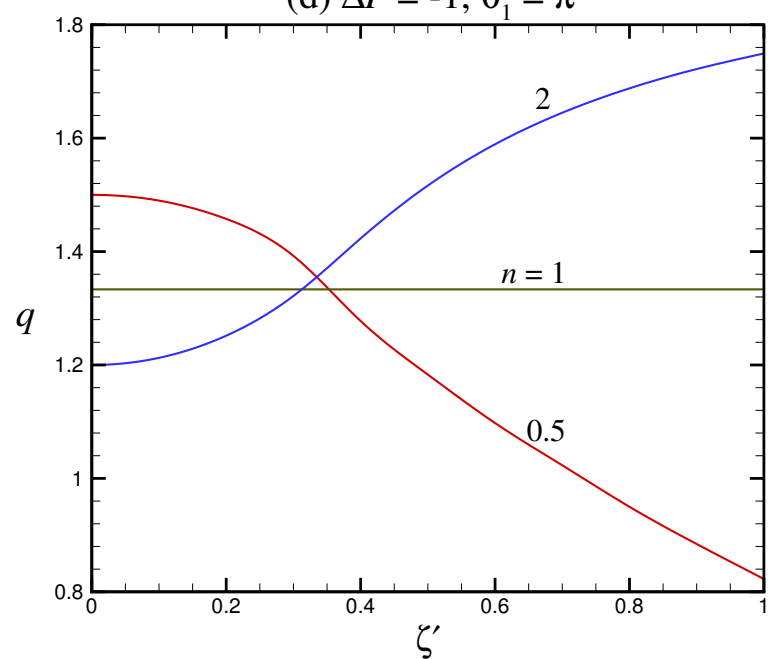

Figure 4: Flow rate $q$ as a function of the amplitude of the zeta potential modulation $\zeta_{1}^{\prime}=$ $\zeta_{2}^{\prime}=\zeta^{\prime}$ for the synchronized wall configuration shown in Fig. 2, where (a) $\Delta P=1, \theta_{1}=0$, (b) $\Delta P=1, \theta_{1}=\pi$, (c) $\Delta P=-1, \theta_{1}=0$, (d) $\Delta P=-1, \theta_{1}=\pi$. 

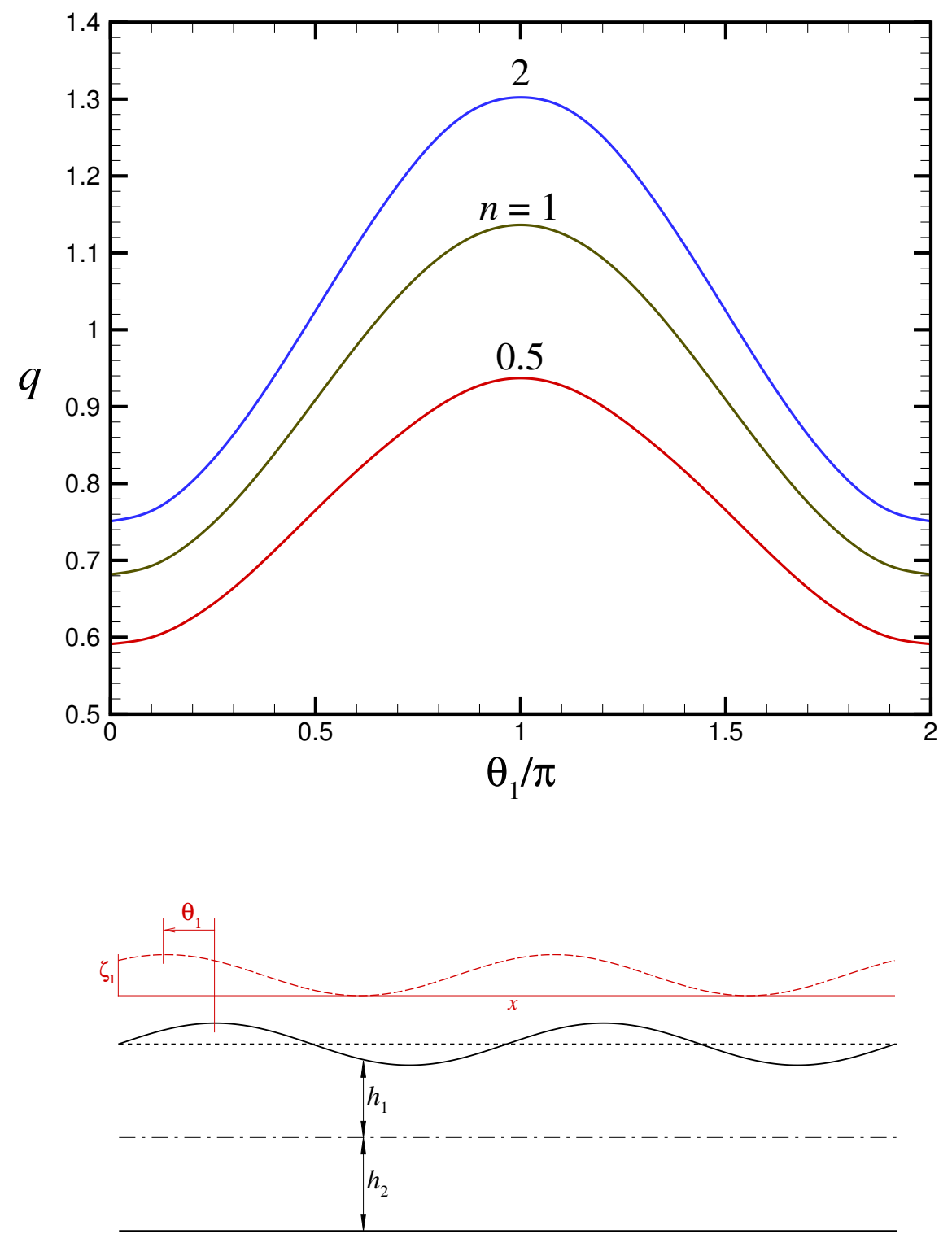

Figure 5: Flow rate $q$ as a function of the phase $\theta_{1}$, where the lower wall of the channel is flat and uncharged: $\bar{\zeta}_{1}=1, \bar{\zeta}_{2}=0, \zeta_{1}^{\prime}=1, \zeta_{2}^{\prime}=0, h_{1}^{\prime}=0.5, h_{2}^{\prime}=0$, and $\Delta P=0$. 

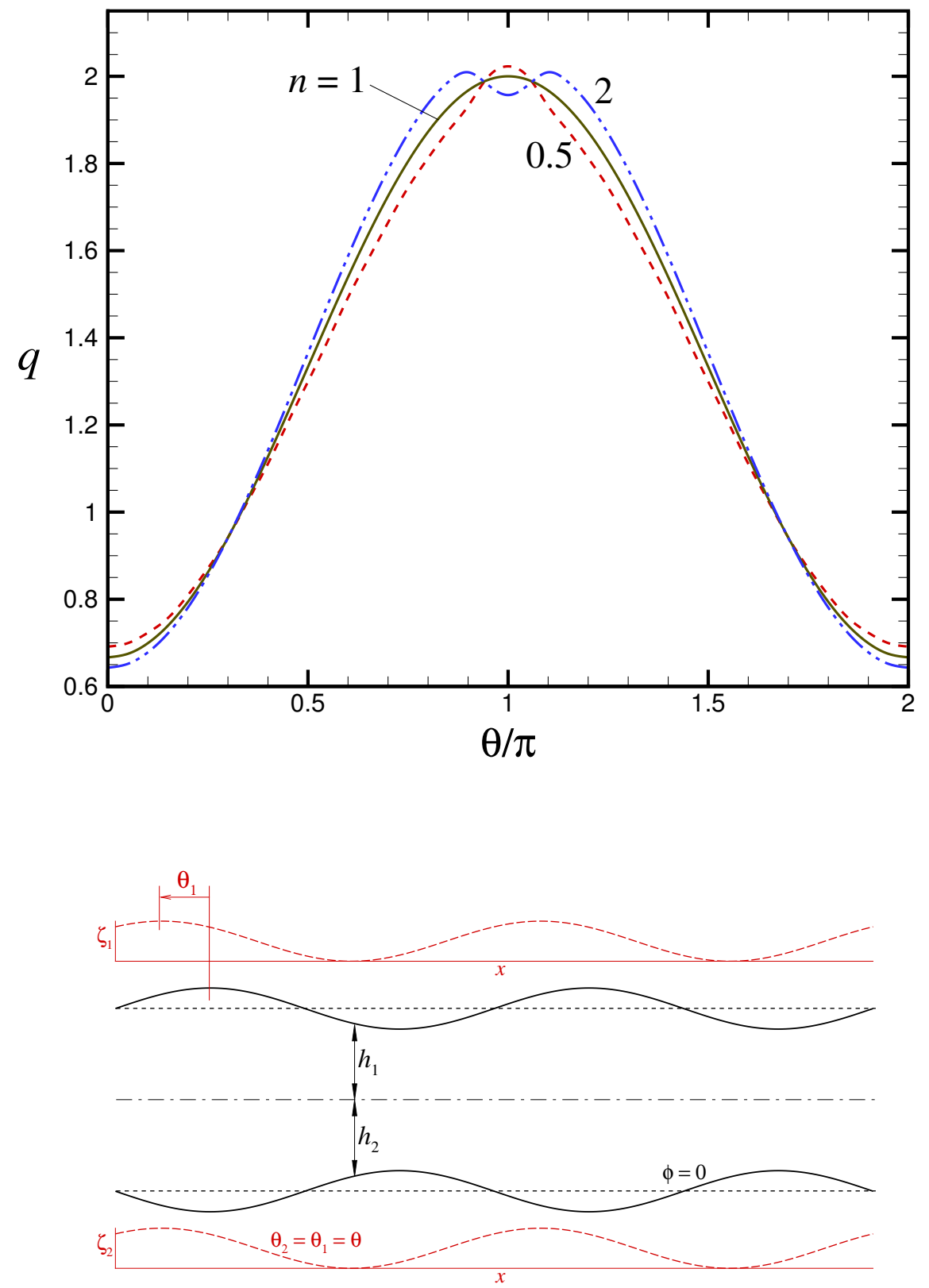

Figure 6: Flow rate $q$ as a function of the phase $\theta_{1}=\theta_{2}=\theta$, where the wall shapes and zeta potentials are symmetrical about the centerline of the channel: $h_{1}^{\prime}=h_{2}^{\prime}=0.5, \phi=0$, $\bar{\zeta}_{1}=\bar{\zeta}_{2}=1, \zeta_{1}^{\prime}=\zeta_{2}^{\prime}=1$, and $\Delta P=0$ 
(a)

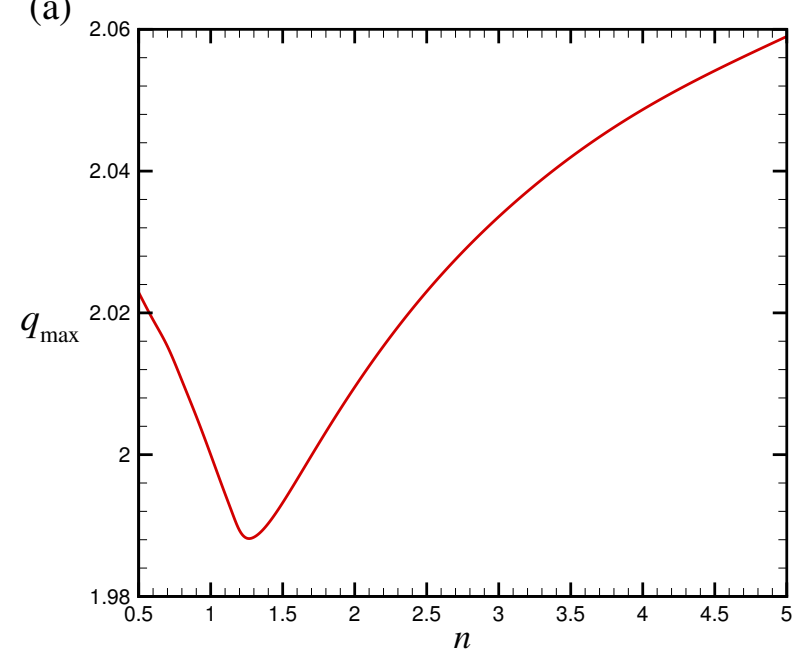

(b)

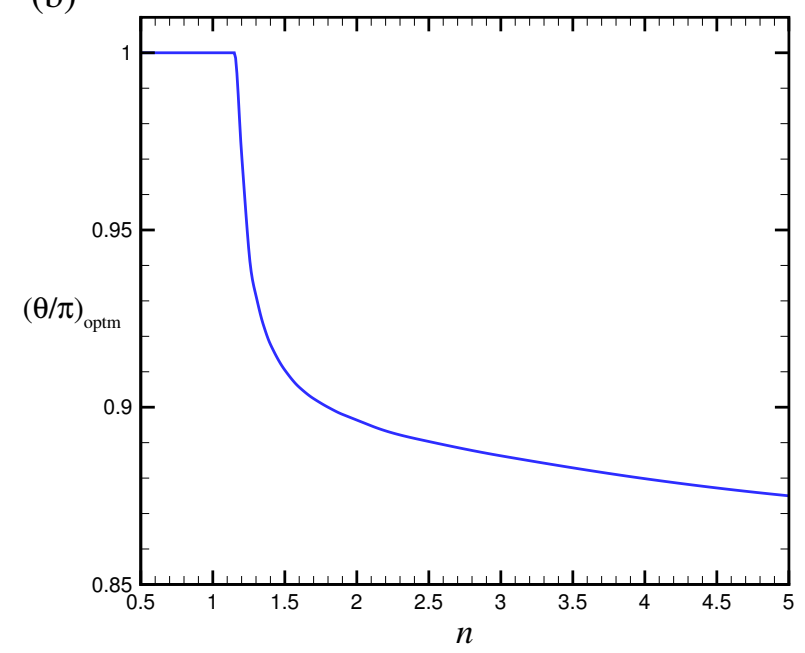

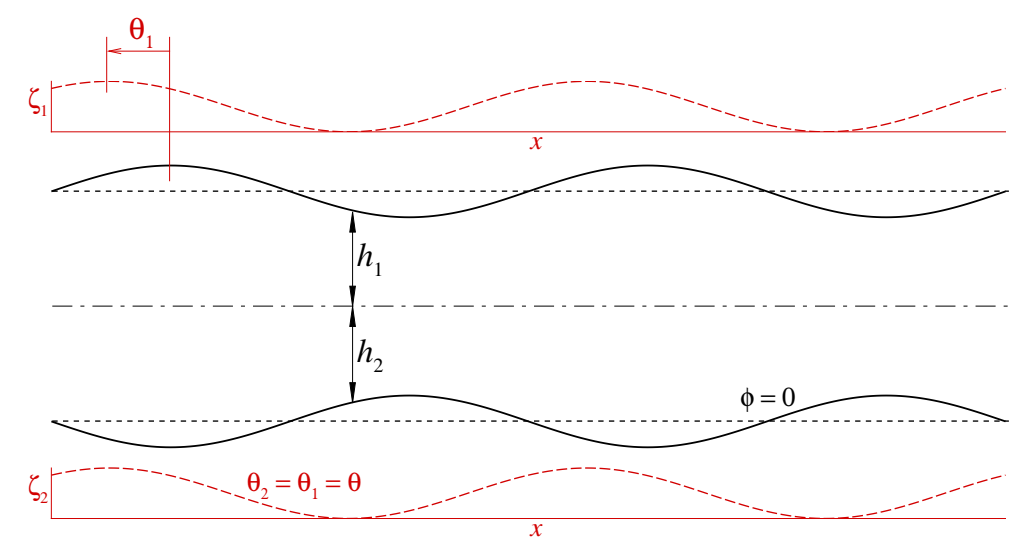

Figure 7: For the symmetrical flow configuration shown in Fig. 5, (a) the maximum flow rate $q_{\max }$, and (b) the corresponding optimum phase $\theta_{\mathrm{optm}}$ as a function of the power-law index $n$. 